\title{
Invasive Fungal Sinusitis in Immunocompromised Patients: A Multicenter, University Hospital Experience in Shiraz
}

\author{
Mohsen Moghadami ${ }^{1}$, Hossein Ruzbahani ${ }^{2}$, Parisa Badiee ${ }^{3 *}$, Abolhassan Faramarzi ${ }^{4}$, \\ Payam Peymani $^{1}$, Kamran Bagheri Lankarani ${ }^{1}$
}

${ }^{1}$ Health Policy Research Center, Shiraz University of Medical Sciences, Shiraz, Iran; ${ }^{2}$ Department of Internal Medicine, Shiraz University of Medical Sciences, Shiraz, Iran; ${ }^{3}$ Alborzi Clinical Microbiology Research Center, Shiraz University of Medical Sciences, Shiraz, Iran; ${ }^{4}$ Department of Otolaryngology, Shiraz University of Medical Sciences, Shiraz, Iran.

Email: peymani.payam@gmail.com

Received September $28^{\text {th }}, 2013$; revised October $28^{\text {th }}, 2013$; accepted November $6^{\text {th }}, 2013$

Copyright (C) 2013 Mohsen Moghadami et al. This is an open access article distributed under the Creative Commons Attribution License, which permits unrestricted use, distribution, and reproduction in any medium, provided the original work is properly cited.

\begin{abstract}
Objective: It is to determine the causes of invasive fungal sinusitis in patients of Shiraz University hospitals, Iran. Methods: This cross-sectional study was conducted during 18 months (from 21 March 2009 till 22 September 2010) in three Shiraz University Hospitals. Thirty-six patients with sings of invasive fungal sinusitis were enrolled, and tissue samples were investigated for histopathology, culture and antifungal susceptibility test. The laboratory results with host factor and sinus computed tomography scan were evaluated for classification of patients as proven, probable and possible invasive fungal sinusitis. Results: Thirty-five patients have involved with at least one risk factor (immune compromised disease, diabetes mellitus, or use of immune suppressed drugs). Radiological findings of parasinus invasion or necrosis were present in 20 patients. By histopathology, 21 patients were considered as proven, from these, 17 samples had positive growth. The culture aetiology agents were 4 Candida, 8 Aspergillus, and 5 Mucor. All positive culture samples were matched with histopathology findings. Significant associations were considered for radiologic finding and histopathology and culture $(p<0.05)$. From 8 patients with mucormycosis histopathology, 6 suffered from diabetes mellitus. None of the antifungal agents were effective on these three types of infections. Conclusion: DM is the most common predisposing factor for IFS followed by ALL and AML. The most common aetiology of IFS was found to be Aspergillus fumigant followed by Mucormycosis and Candida. None of antifungal agents could successfully cover all the species.
\end{abstract}

Keywords: Fungal Sinusitis; Mucor Mycosis; Aspergillus; Invasive Fungal

\section{Introduction}

Opportunistic fungal infections are usually results of immunosuppression and immunodeficiency. Currently several etiologies of immunosuppression lead to increase prevalence of invasive fungal infection (IFI) including leukemia, diabetes mellitus (DM), AIDS, solid organ transplantation, bone marrow transplantation and chemotherapy [1]. All these conditions result in neutropenia which should be treated with wide-spectrum antibiotics. IFI is considered as an important complication of neutropenia which is suspected with persistent fever for 72 - 96 hours after treatment with broad-spectrum antibacterial

\footnotetext{
"Corresponding author.
}

antibiotic [1-3]. However, since the culture methods are insensitive and radiologic findings are nonspecific, the diagnosis of invasive fungal infection remains a challenge [4]. An international consensus on the diagnosis of opportunistic invasive fungal infections in immunocompromised patients with cancer and hematopoietic stem cell transplants was established by the Invasive Fungal Infections Cooperative Group of the European Organization for Research and Treatment of Cancer (EORTC) and the Mycoses Study Group of the National Institute of Allergy and Infectious Diseases (MSG-NIAID) [5,6]. Delay in the treatment of invasive fungal infection during neutropenia causes high mortality in patients with transplants and hematological malignancy $[4,5,7]$. The uncertainty in 
disease diagnosis results in under- or overtreatment of invasive fungal infection. Despite the availability of several new antifungal agents, including triazoles and echinocandins, the effectiveness of antifungal therapy remains uncertain and the effectiveness of neutrophil recovery may not be sufficient if the recovering neutrophils are dysfunctional [8].

Invasive fungal sinusitis (IFS) is a rare disease largely attributable to Aspergillus and Mucor in patients with stem cell transplants and hematological disease [9]. Though the mortality of IFS in immunocompromised patients ranges from $50 \%$ to $80 \%$ [7,9,10], early physical findings are nonspecific and ambiguous (i.e., nasal obstruction, purulent discharge, and epistaxis). Water's view plain radiographs do not distinguish invasive fungal sinusitis from chronic allergic sinusitis. Bony erosion and tissue destruction are often found only in the advanced stage by computed tomography [11,12]. Recent introduction of serial Aspergillus galactomannan antigen test may provide early evidence of IFS. As data regarding this issue are scarce in our region of southern Iran, we performed this study to determine the epidemiology of IFS and the drug sensitivities of the etiological factors in Shiraz, southern Iran.

\section{Materials and Methods}

\subsection{Study Population}

This was a cross-sectional study being performed in Iran during a 1.5-year period from 21 March 2009 till 22 September 2010 including 36 immunosuppressed patients who were further diagnosed to have IFS. Patients with solid organ transplant, hematopoietic stem cell transplants, hematological disease (including severe aplastic anemia, pure red cell aplasia, and hematological malignancy), AIDS and sinusitis diagnosed during their hospital stay were enrolled in this study. Demographic features, history of bone marrow transplant, history of neutropenia (ANC $<500 / \mathrm{mL}$ ), hematological disease status, underlying medical diseases, prolonged corticosteroid therapy ( $>3$ weeks), receiving nucleoside analogue and T-cell suppressor during the previous 3 months and congenital immunodeficiencies were recorded in a questionnaire. The study protocol was approved by the institutional review board of Shiraz University of Medical Sciences and all required patients provided their informed written consents.

\subsection{Sinusitis Diagnosis}

During the study period, those immunosuppressed patients who developed sinusitis were routinely underwent sinus X-ray evaluation and afterwards, otolaryngologist was consulted for focal evaluation and tissue culture. CT and MRI sinus study, surgical biopsy and debridement were performed according to clinicians' decision.
IFS was diagnosed according to EORTC/MSG-NIAID consensus criteria in 2008 [5,6]. The host factors included prolonged neutropenia ( $<500$ neutrophils $/ \mathrm{mm}^{3}$ for $>10$ days) temporally related to the onset of fungal disease, receipt of an allogeneic stem cell transplant, prolonged use of corticosteroids, immunosuppressive agents, or nucleoside analogues during the past 90 days. The clinical criteria included imaging showing sinusitis plus at least one of the following three signs: acute localized pain, nasal ulcer with black eschar, extension from the paranasal sinus across the bony barrier. The microbiological criteria included culture or isolation of fungus from surgical material or sinus aspirate samples, and detection of Aspergillus galactomannan antigens in serum. All the samples were cultured in Sabouraud Dextrose Agar media and sensitivity to amphotericin B 1, capsofungin acetate, voriconazole, itraconazole, ketokonazol were further examined.

\subsection{Proven, Probable, and Possible Invasive Fungal Sinusitis}

Proven, probable, and possible IFS were defined mainly according to the EORTC/MSG-NIAID criteria [5,6]. Proven IFS was defined by the presence of fungi associated tissue damage on histopathologic examination of a biopsy specimen; or positive culture result, consistent with infection, from a sample obtained aseptically from a clinically or radiologically abnormal site. Probable IFS was defined by the presence of at least one host factor criterion, one microbiological criterion, and one clinical criterion. Possible IFS was defined by the presence of at least one host factor criterion and one clinical criterion.

\subsection{Statistical Analysis}

Statistical analyses were performed using the SPSS software, version 16.0 (SPSS Inc., Chicago, Ill., USA). The chi-square test was used to compare the proportions between groups. The results are expressed as mean \pm SD and proportions as appropriated. A two-tailed p-value less than 0.05 was considered statistically significant.

\section{Results}

Overall we included 36 patients among whom there were 15 (41.6\%) man and 21 (58.4\%) women. The mean age of the patients was 40 years with maximum age of 70 years. Sixteen $(44 \%)$ of the patients were diagnosed to have DM. Thirty patients had at least one co-morbidities or sinus destruction. The most common predisposing condition was ALL in 5 and AML in 5 followed by sinus surgery in 3 , CML in 3 , CLL in 1 , major $\beta$-thalassemia in 2 , aplastic anemia in 1, Hodgkin's lymphoma in 1, nonHodgkin's lymphoma in 2 and other diseases (congenital 
immmunodeficiencies, polycythemia vera) in 7 . Bone marrow transplantation was performed in $19.4 \%$ of patients. Overall, $47.2 \%$ received immunosuppressant and $41.7 \%$ received cytotoxic agents. Twenty-three patients were on anti-fungal treatment at time of inclusion in the study. Regarding clinical signs, 31 (86.1\%) had nasal congestion while 30 (83.3\%) had facial pain and 18 (50\%) had epistaxis. Ocular involvement was reported in 9 patients and 11 patients had neurological involvement. Unilateral involvement of VII nerve and bilateral involvement of III were the most common neurological complications. Radiological signs of sinus invasion were reported in 20 (55.6\%) patients out of which 18 (50\%) had clinical signs of nasal, orbital or palate involvement. Severe neutropenia was reported in 11 (30.6\%) of patients (Table 1).

Overall 21 samples tested positive for $\mathrm{KOH}$ including 1 Yeast and Boding Yeast, 3 Pseudo Hyphae, 9 Septated Hyphae and 8 Nonseptated Hyphae. However 16 samples had positive cultures including 4 Candida (2 Candida albicans, 1 Candida glabrata and 1 Candida krusei), 6 Aspergillus flavus, 1 Aspergillus fumigates and 5 Mucormycosis. The culture results had $100 \%$ concordance with $\mathrm{KOH}$ results (Table 2). Posaconazole and Fluconazole were found to have intermediate sensitivity (SDD). This means that the resistance can be overcome by increase in dose. Mucormycosis infection was significantly associated with DM $(p<0.05)$. Fifteen out of 18 patients with signs of local invasion were further found to be proven infection $(p<0.05)$. The most common aetiology of IFS was found to be Aspergillus fumigates followed by Mucormycosis and Candida. None of antifungal could successfully cover all the species. Voriconazole was found to be appropriate for treatment of Aspergillus and Candida while Amphotericin B was found to be appropriate for treatment of Mucormycosis. Posaconazole was found to be effective on both Aspergillus and Mucormycosis.

\section{Discussion}

In most developing countries, increased prevalence of resistant fungal infections and lack of appropriate diagnostic facilities is an important health issue. In addition, the number of patients suffering from immunodeficiencies is increasing dramatically in these countries. In this study we found that DM is the most common predisposing factor for IFS followed by ALL and AML. The most common aetiology of IFS was found to be Aspergillus fumigates followed by Mucormycosis and Candida. Chen and colleagues [13] demonstrated that IFS occurred in $1.77 \%$ of hospitalized patients with hematological disorders. IFS caused significantly higher mortality in AML patients with prolonged neutropenia (>10 days). IFS developed more frequently in patients with AML, myelodysplastic syndrome, and aplastic anemia, but not at all in patients with lymphoma/myeloma. In the literature review, most patients with lymphoma who developed IFS are recipients of myeloablative allogeneic stem cell transplants [14-18]. Compared with other subtypes of hematological malignancy, patients with AML have significantly higher risk of IFS. The risk of developing IFS in AML relates to neutropenia and less to the intensity of chemotherapy regimens.

Prolonged neutropenia in patients with myeloid malignancies may contribute to underlying disease, intensity and dosage of chemotherapy, colony-stimulating factor, and concurrent medication. Invasive mold infection often occurs when a large burden of spores from an environmental source is deposited on mucosal membranes lacking an effective phagocytic host defense [19]. Using cytokine growth factors to decrease the period of chemotherapy-associated neutropenia and using laminar air flow rooms for protection against IFS [20] may reduce the risk of IFS after allogeneic stem cell transplantation [21].

The clinical mycological spectrum of IFS is limited in patients with stem cell transplants and hematological disease [14-18]. Aspergillus and Mucor are the main mold found in biopsy, however, the prevalence is highly variable in different geographic regions [14-18,22,23]. Chen and colleagues [13] founded that Aspergillus flavus (44\%) was the most common isolate which is in concordance with our study. Aspergillus flavus, with its unique ability to survive at higher temperatures, is the predominant pathogen in countries, including most of the Middle East,

Table 1. Clinical and host factors of local invasion in 36 patients suffering from invasive fungal sinusitis (IFS).

\begin{tabular}{cccccc}
\hline & Number & Admission in previous 3 months & DM & Radiological signs of local invasion & Tissue necrosis signs \\
\hline Proven & 21 & 12 & 12 & 18 & 15 \\
Probable & 4 & 1 & 2 & 1 & 2 \\
Possible & 7 & 2 & 1 & 0 & 0 \\
No IFS & 4 & 2 & 1 & 1 & 1 \\
ALL & 36 & 17 & 16 & 20 & 18 \\
\hline
\end{tabular}


Table 2. Culture and antibiogram results of $\mathbf{3 6}$ patients suffering from invasive fungal sinusitis (IFS).

\begin{tabular}{|c|c|c|c|c|c|c|}
\hline Organism & Antifungal & Resistant & Sensitive & Range & MIC 50\% & MIC $90 \%$ \\
\hline \multirow{6}{*}{$\begin{array}{l}\text { Aspergillus } \\
\quad(n=7)\end{array}$} & Amphotericin B & 5 & 3 & $0.5-16$ & 0.75 & 16 \\
\hline & Ketoconazole & 0 & 8 & $0.5-3$ & 1 & 3 \\
\hline & Itraconazole & 2 & 6 & $0.047-32$ & 0.125 & 32 \\
\hline & Posaconazole & 0 & 8 & $0.047-0.19$ & 0.19 & 0.19 \\
\hline & Caspofungin & 0 & 8 & $0.32-0.5$ & 0.094 & 0.75 \\
\hline & Voriconazole & 0 & 8 & $0.94-0.5$ & 0.19 & 0.5 \\
\hline \multirow{6}{*}{$\begin{array}{l}\text { Mucormycosis } \\
(n=5)\end{array}$} & Amphotericin B & 0 & 5 & $0.063-0.75$ & 0.125 & 0.75 \\
\hline & Ketoconazole & 1 & 4 & $0.25-4$ & 0.25 & 4 \\
\hline & Itraconazole & 4 & 1 & $0.29-64$ & 0.29 & 64 \\
\hline & Posaconazole & 0 & 5 & $0.75-2$ & 0.75 & 2 \\
\hline & Caspofungin & 4 & 1 & $0.14-32$ & 4 & 32 \\
\hline & Voriconazole & 3 & 2 & $1.15-16$ & 1.25 & 16 \\
\hline \multirow{8}{*}{$\begin{array}{l}\text { Candida } \\
(n=4)\end{array}$} & Amphotericin B & 0 & 4 & $0.5-1$ & 0.75 & 1 \\
\hline & Ketoconazole & 1 & 3 & $0.125-32$ & 1 & 32 \\
\hline & Itraconazole & 3 & 1 & $0.032-32$ & 2.5 & 32 \\
\hline & Posaconazole & 2 & 2 & $0.094-32$ & 0.25 & 32 \\
\hline & Caspofungin & 0 & 4 & $0.125-0.29$ & 0.125 & 0.29 \\
\hline & Voriconazole & 0 & 4 & $0.032-0.75$ & 0.25 & 0.75 \\
\hline & Nystatin & 0 & 4 & $4.6-9.25$ & 4.6 & 9.25 \\
\hline & Fluconagole & 1 & 3 & $0.25-64$ & 24 & 64 \\
\hline \multirow{6}{*}{$\begin{array}{c}\text { Total } \\
(n=16)\end{array}$} & Amphotericin B & 5 & 12 & $0.063-61$ & 0.5 & 4 \\
\hline & Ketoconazole & 2 & 15 & $0.125-32$ & 1 & 2 \\
\hline & Itraconazole & 9 & 8 & $0.032-64$ & 0.29 & 32 \\
\hline & Posaconazole & 2 & 15 & $0.047-32$ & 0.19 & 2 \\
\hline & Caspofungin & 4 & 13 & $0.125-32$ & 0.125 & 4 \\
\hline & Voriconazole & 3 & 14 & $0.032-16$ & 0.25 & 2 \\
\hline
\end{tabular}

Africa, and Southeast Asia [24,25]. Rare IFS in Asia and Africa were reported, the clinical response varies differently with fungal subtypes, and further epidemiology study should be investigated. Mucormycosis is an emerging cause of IFS with a rapid fatal course in patients with hematological disorders [26,27]. Effective treatment for Mucormycosis should be investigated.

The symptoms and signs of paranasal sinusitis (such as nasal discharge, stuffiness, epistaxis, periorbital swelling, and maxillary tenderness) are nonspecific for IFS [28]. Symptoms and signs such as nose ulceration, eschar of the nasal mucosa, black necrotic lesions, and perforation of the hard palate are more specific, but these findings are present only at an advanced stage [29]. The use of CT and MRI in the diagnosis of invasive fungal sinusitis has been reported [12]. Diagnostic radiological evidence of invasive fungal sinusitis includes erosion of sinus walls, extension of infection to neighboring structures, and extensive skull base destruction. However, most patients do not have classic findings in the early phase of invasive fungal sinusitis. Earlier diagnosis by using serial Aspergillus galactomannan antigen test in the modern medical era to detect IFS, may lead to early introduce anti-fungal agent and surgical debridement, and potentially decreas- 
ed morbidity and mortality in high risk patients.

\section{Conclusion}

In conclusion, DM is the most common predisposing factor for IFS followed by ALL and AML. The most common aetiology of IFS was found to be Aspergillus fumigant followed by Mucormycosis and Candida. None of antifungal agents could successfully cover all the species.

\section{Acknowledgments}

This research project was financially supported by Health Policy Research Center affiliated with Shiraz University of Medical Sciences. Also, there is no conflict of interest to be declared regarding this manuscript.

\section{REFERENCES}

[1] J. Crawford, D. C. Dale and G. H. Lyman, "Chemotherapy-Induced Neutropenia: Risks, Consequences, and New Directions for Its Management," Cancer, Vol. 100, 2004, pp. 228-237. http://dx.doi.org/10.1002/cncr.11882

[2] S. Vento and F. Cainelli, "Infections in Patients with Cancer Undergoing Chemotherapy: Etiology, Prevention, and Treatment," Lancet Oncology, Vol. 4, 2003, pp. 595-604. http://dx.doi.org/10.1016/S1470-2045(03)01218-X

[3] E. J. Bow, "Management of the Febrile Neutropenic Cancer Patient: Lessons from 40 Years of Study," Clin Microbiol Infect, Vol. 11, No. S5, 2005, pp. 24-29. http://dx.doi.org/10.1111/j.1469-0691.2005.01240.x

[4] N. G. Almyroudis and B. H. Segal, "Prevention and Treatment of Invasive Fungal Diseases in Neutropenic Patients," Current Opinion in Infectious Diseases, Vol. 22, 2009, pp. 385-393. http://dx.doi.org/10.1097/QCO.0b013e32832e074d

[5] S. Ascioglu, J. H. Rex, B. de Pauw, J. E. Bennett, J. Bille, F. Crokaert, et al., "Invasive Fungal Infections Cooperative Group of the European Organization for Research and Treatment of Cancer; Mycoses Study Group of the National Institute of Allergy and Infectious Diseases. Defining Opportunistic Invasive Fungal Infections in Immunocompromised Patients with Cancer and Hematopoietic Stem Cell Transplants: An International Consensus," Clinical Infectious Diseases, Vol. 34, 2002, pp. 7-14. http://dx.doi.org/10.1086/323335

[6] B. De Pauw, T. J. Walsh, J. P. Donnelly, D. A. Stevens, J. E. Edwards, T. Calandra, et al., "European Organization for Research and Treatment of Cancer/Invasive Fungal Infections Cooperative Group; National Institute of Allergy and Infectious Diseases Mycoses Study Group (EORTC/ MSG) Consensus Group. Revised Definitions of Invasive Fungal Disease from the European Organization for Research and Treatment of Cancer/Invasive Fungal Infections Cooperative Group and the National Institute of Allergy and Infectious Diseases Mycoses Study Group (EORTC/MSG) Consensus Group," Clinical Infectious Diseases, Vol. 46, 2008, pp. 1813-1821. http://dx.doi.org/10.1086/588660

[7] R. Martino and M. Subira, "Invasive Fungal Infections in Hematology: New Trends," Annals of Hematology, Vol. 81, 2002, pp. 233-243. http://dx.doi.org/10.1007/s00277-002-0466-3

[8] E. J. Anaissie, "Diagnosis and Therapy of Fungal Infection in Patients with Leukemia-New Drugs and Immunotherapy,” Best Practice \& Research Clinical Haematology, Vol. 21, 2008, pp. 683-690.

http://dx.doi.org/10.1016/j.beha.2008.10.001

[9] R. D. deShazo, K. Chapin and R. E. Swain, "Fungal Sinusitis,” The New England Journal of Medicine, Vol. 337, 1997, pp. 254-259. http://dx.doi.org/10.1056/NEJM199707243370407

[10] S. L. Parikh, G. Venkatraman and J. M. DelGaudio, "Invasive Fungal Sinusitis: A 15-Year Review from a Single Institution,” American Journal of Rhinology, Vol. 18, 2004, pp. 75-81.

[11] W. T. Anselmo-Lima, R. P. Lopes, F. C. Valera and R. C. Demarco, "Invasive Fungal Rhinosinusitis in Immunocompromised Patients,” Rhinology, Vol. 42, 2004, pp. 141-144.

[12] R. C. Howells and H. H. Ramadan, "Usefulness of Computed Tomography and Magnetic Resonance in Fulminant Invasive Fungal Rhinosinusitis," American Journal of Rhinology, Vol. 15, 2001, pp. 255-261.

[13] C. Y. Chen, W. H. Sheng, A. Cheng, Y. C. Chen, W. Tsay, J. L. Tang, et al., "Invasive Fungal Sinusitis in Patients with Hematological Malignancy: 15 Years Experience in a Single University Hospital in Taiwan,” BMC Infect Disease, Vol. 11, 2011, p. 250. http://dx.doi.org/10.1186/1471-2334-11-250

[14] P. C. Iwen, M. E. Rupp and S. H. Hinrichs, "Invasive Mold Sinusitis: 17 Cases in Immunocompromised Patients and Review of the Literature," Clinical Infectious Diseases, Vol. 24, 1997, pp. 1178-1184. http://dx.doi.org/10.1086/513662

[15] P. E. Drakos, A. Nagler, R. Or, E. Naparstek, J. Kapelushnik, D. Engelhard, G. Rahav, D. Ne'emean and S. Slavin, "Invasive Fungal Sinusitis in Patients Undergoing Bone Marrow Transplantation,” Bone Marrow Transplant, Vol. 12, 1993, pp. 203-208.

[16] D. Saah, P. E. Drakos, J. Elidan, I. Braverman, R. Or and A. Nagler, "Rhinocerebral Aspergillosis in Patients Undergoing Bone Marrow Transplantation,” Annals of Otology, Rhinology, and Laryngology, Vol. 103, 1994, pp. 306-310.

[17] G. H. Talbot, A. Huang and M. Provencher, "Invasive Aspergillus Rhinosinusitis in Patients with Acute Leukemia," Reviews of Infectious Diseases, Vol. 13, 1991, pp. 219-232. http://dx.doi.org/10.1093/clinids/13.2.219

[18] R. S. Weber and G. Lopez-Berestein, "Treatment of Invasive Aspergillus Sinusitis with Liposomal-Amphotericin B,” Laryngoscope, Vol. 97, 1987, pp. 937-941. http://dx.doi.org/10.1288/00005537-198708000-00010

[19] A. Wald, W. Leisenring, J. A. van Burik and R. A. Bowden, "Epidemiology of Aspergillus Infections in a Large 
Cohort of Patients Undergoing Bone Marrow Transplantation," The Journal of Infectious Diseases, Vol. 175, 1997, pp. 1459-1466. http://dx.doi.org/10.1086/516480

[20] M. Ruhnke, A. Böhme, D. Buchheidt, K. Donhuijsen, H. Einsele, R. Enzensberger, et al., "Infectious Diseases Working Party (AGIHO) of the German Society of Hematology and Oncology (DGHO) Diagnosis of Invasive Fungal Infections in Hematology and Oncology_Guidelines of the Infectious Diseases Working Party (AGIHO) of the German Society of Hematology and Oncology (DGHO)," Annals of Hematology, Vol. 82, No. S2, 2003, pp. S141-S148. http://dx.doi.org/10.1007/s00277-003-0768-0

[21] D. C. Lanza, H. J. Dhong, P. Tantilipikorn, J. Tanabodee, D. M. Nadel and D. W. Kennedy, "Fungus and Chronic Rhinosinusitis: From Bench to Clinical Understanding," Annals of Otology, Rhinology, and Laryngology, Vol. 196, No. S1, 2006, pp. 27-34.

[22] Y. Li, Y. Li, P. Li and G. Zhang, "Diagnosis and Endoscopic Surgery of Chronic Invasive Fungal Rhinosinusitis," American Journal of Rhinology and Allergy, Vol. 23, 2009, pp. 622-625. http://dx.doi.org/10.2500/ajra.2009.23.3361

[23] A. P. Ingley, S. L. Parikh and J. M. DelGaudio, “Orbital and Cranial Nerve Presentations and Sequelae Are Hallmarks of Invasive Fungal Sinusitis Caused by Mucor in Contrast to Aspergillus,” American Journal of Rhinology, Vol. 22, 2008, pp. 155-158. http://dx.doi.org/10.2500/ajr.2008.22.3141
[24] S. Krishnan, E. K. Manavathu and P. H. Chandrasekar, "Aspergillus Flavus: An Emerging Non-Fumigatus Aspergillus Species of Significance,” Mycoses, Vol. 52, 2009, pp. 206-222.

http://dx.doi.org/10.1111/j.1439-0507.2008.01642.x

[25] G. Surya Prakash Rao, S. B. Mann, P. Talwar and M. M. Arora, "Primary Mycotic Infection of Paranasal Sinuses," Mycopathologia, Vol. 84, 1984, pp. 73-76. http://dx.doi.org/10.1007/BF00436515

[26] W. A. Bethge, M. Schmalzing, G. Stuhler, U. Schumacher, S. M. Kröber, M. Horger, H. Einsele, L. Kanz and H. Hebart, "Mucormycoses in Patients with Hematologic Malignancies: An Emerging Fungal Infection,” Haematologica, Vol. 90, No. S1, 2005, p. ECR22.

[27] A. Nosari, P. Oreste, M. Montillo, G. Carrafiello, M. Draisci, G. Muti, et al., "Mucormycosis in Hematologic Malignancies: An Emerging Fungal Infection,” Haematologica, Vol. 85, 2000, pp. 1068-1071.

[28] A. E. Suslu, O. Ogretmenoglu, N. Suslu, O. T. Yucel and T. M. Onerci, "Acute Invasive Fungal Rhinosinusitis: Our Experience with 19 Patients,” European Archives of Otorhinolaryngology, Vol. 266, 2009, pp. 77-82. http://dx.doi.org/10.1007/s00405-008-0694-9

[29] R. Y. Hachem, M. R. Boktour, H. A. Hanna, R. Husni, E. Y. Hanna, X. Keutgen, et al., "Sinus Surgery Combined with Antifungal Therapy Is Effective in the Treatment of Invasive Aspergillus Sinusitis in Neutropenic Patients with Cancer,” Infection, Vol. 36, 2008, pp. 539-542. http://dx.doi.org/10.1007/s15010-008-7388-5 